\title{
The insulin dilemma in resource-limited countries. A way forward?
}

\author{
G. V. Gill • J. S. Yudkin $\cdot$ H. Keen $\cdot$ D. Beran
}

Received: 5 August 2010/Accepted: 9 August 2010/Published online: 12 September 2010

(C) Springer-Verlag 2010

\begin{abstract}
The International Insulin Foundation (IIF) has developed and validated a needs-assessment instrument called the Rapid Assessment Protocol for Insulin Access (RAPIA) which has been used in seven countries in four continents to analyse the constraints to delivering effective continuing care for people with diabetes. One major contributor to the difficulties in availability of insulin is a failure to use the least costly sources and types of insulin and other effective drugs for diabetes. The purchase of insulins can consume as much as $10 \%$ of government expenditure on drugs, this being highly sensitive to the selection of newer analogue insulins as first-choice options, which cost between three and 13 times more than biosynthetic human insulin. Insulin cartridges for use with injection pens further add to costs. Similar considerations apply to most of the newer treatments for people with type 2 diabetes, which may cost up to 40 times more than metformin and sulfonylureas, still considered first-line drugs by European and US guidelines. Both biosynthetic human insulin and the first-line oral hypoglycaemic drugs are available from generic manufacturers. With the present price differentials, there is thus a growing need for countries involved in tendering for sourcing insulin to be provided with the guarantees of Good Manufacturing Practice, quality and bioequivalence, which would come from a WHO Pre-Qualification Scheme as currently exists for a variety of drugs for chronic diseases, both communicable and non-communicable. The IIF has developed a
\end{abstract}

G. V. Gill $(\bowtie) \cdot$ J. S. Yudkin $\cdot$ H. Keen $\cdot$ D. Beran

International Insulin Foundation,

Centre for International Health and Development,

Institute for Child Health,

30 Guilford Street,

London, UK

e-mail: g.gill@liv.ac.uk position statement on the provision and choice of diabetes treatments in resource-limited settings which should be applicable wherever consideration of resources is a component of therapeutic decision making.

Keywords Analogue insulins - Diabetes drug costs . Diabetes healthcare $\cdot$ Insulin regimens $\cdot$ Low- and middle-income countries . Needs assessment .

Oral hypoglycaemic agents
Abbreviations
IIF International Insulin Foundation
RAPIA Rapid Assessment Protocol for Insulin Access

\section{Introduction}

Insulin is regarded as a relatively inexpensive drug by adequately-funded health services of Western countries. In resource-limited countries, however, this is not the case. Such countries are mainly tropical (most are in sub-Saharan Africa), but some are in Eastern Europe, Asia and South America. Here, insulin is expensive relative to the total healthcare budget and has to compete with other demands, in particular, for anti-retroviral drugs. The same is true of self-monitoring blood glucose meters and strips, $\mathrm{HbA}_{1 \mathrm{c}}$ assays, newer agents to treat type 2 diabetes, and adequate provision of trained diabetes healthcare professionals. Moreover, the increasing financial pressures on healthcare units and systems in industrialised countries has led to a recognition that the costs, as well as the comparative effectiveness, of newer agents must be taken into account, with the consequent proliferation of essential drugs lists and generic purchasing. 
As long ago as 1992, Chale and colleagues in Tanzania estimated that the insulin-treated diabetic population of their country (comprising $0.2 \%$ of the total) was using $8 \%$ of the government healthcare budget [1]. In 1994, this problem was termed the 'insulin dilemma' [2] and it has been much discussed and widely accepted [3-7]. More recent surveys have confirmed the breadth of the problem $[8,9]$, and there is no doubt that deaths owing to diabetic ketoacidosis as a result of insulin shortage occur in people with type 1 diabetes living in remote, resource-poor areas [10]. Chronic self-underdosing with insulin also occurs, to economise and to make supplies last longer in case of interruption. The consequences of this are chronic hyperglycaemia, accelerated complication risk, stunted growth in children and adolescents, low life quality and early death.

\section{The International Insulin Foundation}

Both the WHO [11] and the International Diabetes Federation [12] have long recognised and publicised insulin shortages in poor countries. An organisation called Insulin for Life also collects unused insulin from adequatelyresourced countries and redistributes it to areas of need [13]. In 2002, the International Insulin Foundation (IIF) was formed by a multinational group of academic and clinical diabetologists, with an aim to improve access to insulin in resource-poor countries. Specifically, universal availability of insulin for those with type 1 diabetes was seen as a basic human right. The IIF also supported access to insulin, when appropriate, for people with type 2 diabetes, and advocated improved availability of diabetes care in general.

With limited funding, the IIF systematically explored current insulin availability in several resource-poor countries, developing a purpose-constructed assessment tool that operates at central, intermediate and local levels. This has become known as the Rapid Assessment Protocol for Insulin Access (RAPIA) [14]. RAPIAs have been carried out in several African countries (Mozambique, Zambia and Mali) [8], as well as more recently in Nicaragua, Vietnam and Kyrgyzstan. In Mozambique, an initial RAPIA opened the way for a highly successful twinning programme with the British charity Diabetes UK [15-17].

The IIF surveys, using the RAPIA protocol, have identified multiple problems leading to inadequate insulin supply at the grass roots level. These include problems with assessment of needs, logistic problems of distribution, poor knowledge at all levels of the health system about insulin and its use, and clear revenue shortages. However, an additional contributor to the difficulties in availability of insulin is a failure to use the least costly but effective sources and types of insulin and other drugs for diabetes.
The insulin dilemma explored in resource-poor settings

Our work in Mozambique showed that $10 \%$ of the government expenditure on medications was being used on insulin $[15,16]$. The most recent RAPIA by the IIF in Kyrgyzstan showed that $57 \%$ of national insulin expenditure (and $42 \%$ of the total budget for diabetes drugs and supplies) was for the purchase of costly newer analogue insulins [18]: the cost of glargine, for example, was 13 times that of brandname isophane insulin. Data from other resource-poor areas of the world also suggests that expenditure on analogue insulin is financially significant, with the price of these insulins generally exceeding that of human insulin by a factor of three to five. The use of animal insulin, and its availability, is now negligible $[19,20]$.

Another factor adding to insulin costs in many countries is the use of insulin pens rather than the traditional (and much cheaper) reusable syringes and needles. Insulin supplied in cartridges for pen use is around two to three times more expensive unit for unit than that supplied in vials for use with syringes. Although insulin injection pens have advantages in terms of convenience of use, particularly in young people and in situations where people may be (or feel) stigmatised by using syringes, these need to be set against the extra costs.

Most of the world's insulin is produced by three major pharmaceutical companies, and the prices of their insulins are higher than those made by a variety of manufacturers in India, Poland, Russia, China, Egypt and elsewhere [21]. Whilst an equity pricing scheme operated by Novo Nordisk has substantially reduced the price of their insulins in the 50 least developed countries, to around US\$4.50 for a $10 \mathrm{ml}$ vial of U100 human insulin, this scheme has not been universally adopted, and in any event applies only to insulin supplied to governments and not to that purchased from private pharmacies.

\section{The analogue paradox and its global relevance}

It is something of a paradox that analogue insulins have, over the last decade, captured such a large proportion of the total insulin market despite there being relatively limited evidence for significant benefit. Three connected themes make the issue of insulin analogues of relevance to the global diabetes community: comparative effectiveness, the marketing strategies of insulin manufacturers, and the global economic downturn. Analogue insulins may have some benefits in individual patients, and trials suggest that long-acting analogues at least may reduce the risk of hypoglycaemia (particularly nocturnal) [22]. A critical analysis of the evidence is, however, less convincingindeed, the title of one review article is interesting (Nice 
insulins, pity about the evidence) [23]. Additionally, an observational report from Johannesburg, described as 'from the coalface', showed no glycaemic improvement in patients moved to a long-acting analogue insulin from isophane preparations, though those patients with frequent hypoglycaemia did report a reduction in rates of hypoglycaemia [24]. In another recent 'real-life' study of 25 type 1 diabetic patients using continuous glucose monitoring, $28 \%$ had episodes of nocturnal hypoglycaemia despite almost two-thirds (64\%) being on long-acting analogue insulins [25]. There is a growing use of comparative effectiveness research to inform the selection of drugs, and considerations of the scanty additional benefits of analogue insulins in relation to additional costs have led national pharmaceutical agencies in some industrialised countries to limit prescribing of or reimbursement for these preparations [26-28].

Despite the marginal benefits of analogue insulins, particularly when considered alongside their substantially higher costs, the last decade has seen a rapid growth in their use in high-income countries. Data on changing patterns of global insulin use over the last decade are available from IMS Health [19, 29]. Despite the limitations of the data available with regard to the countries represented, and lack of an overall representation of the insulin market (the data for low-income countries are of retail rather than government sales), there are three clear trends [19]. First, over the last decade there has been a decline in animal insulin use in all regions of the world, so that currently less than $25 \%$ of countries in all regions use any animal insulin at all.

The second trend is that use of human insulin has declined from being the sole type of insulin used to representing about one-third in high-income countries and two-thirds in middle-income countries by 2009. In lowincome countries, this decline started later than elsewhere, with human insulin still comprising over $94 \%$ of all insulin in all the surveyed countries. Finally, decreasing proportionate use of human insulin in countries surveyed by IMS was mirrored by rising proportions of analogue insulin, increasing to some two-thirds of all insulin in high income countries by 2009 , with middle- and low-middle-income country trends following behind. In low-income countries, however, analogue insulin still represented a median of only $4 \%$ of insulin usage by 2009 .

A similar trend has been shown in work by Currie et al. [30] who reported that, in 2000, $2.9 \%$ of insulin dispensed in the UK was animal, $86.3 \%$ human and $10.7 \%$ analogue. By 2008 , however, the use of human insulin had fallen to $23.2 \%$, with analogues representing $76.1 \%$ of the total. By this stage, animal insulin represented only $0.7 \%$ of the market.

In summary, these data show the evolution of an international insulin market in which animal insulin is first replaced by human insulin and then human insulin replaced by analogue. There is clearly also a delay in this shift between income levels, with it occurring first in high-income countries, about 4 years later in upper middle-income countries and about 2 years later in lower middle-income countries. This shift is not yet visible in the lowest income countries, but results from the IIF's work in Kyrgyzstan show that this worrying trend has started.

The marketing of analogue insulins, with their injection devices and cartridges, dominates the commercial considerations of the major insulin suppliers, and this has already led to their discontinuing production of several brands of human insulin or the availability of these brands in vials. NovoNordisk is withdrawing its human soluble-NPH 30:70 insulin from the UK market by the end of 2010. There is substantial concern in many resource-poor countries that this trend will accelerate, with the production of cheaper human insulin being halted in a similar fashion to that of animal insulins when recombinant human insulin began to dominate the market.

There has, however, been a major change in the patterns of insulin manufacture during the last 20 years, with the entry of numerous producers of recombinant human (and animal) insulin around the world. Although these companies are largely targeting domestic markets, the nature of recombinant technology readily allows for increasing production. In many countries, including some in Europe and North America, national purchasing agencies or individual healthcare organisations are using a tendering process to purchase quality-assured generic recombinant human insulin. This approach would be greatly facilitated by the attesting of quality and bioequivalence, which would come from a Prequalification Scheme [31] of the type which the WHO operates for some drugs, and would permit the independence of supplies of recombinant human insulin from the commercial considerations of the major producers. Such an approach might help reduce the costs of initiating insulin treatment for newly treated patients and lessen the financial burden to individual and state in countries affected by the global economic downturn.

\section{Potential solutions?}

Overall, we believe that present cost-benefit considerations do not support the general use of analogue insulins, particularly in resource-poor countries and settings, although they may be indicated in particular situations if resources permit. Simple human (or animal) insulins, if properly used, will nearly always suffice. Pen injectors are very expensive, and benefits from their use must be carefully considered against alternative uses of resources, particularly when basic insulin access and availability cannot be guaranteed. Cartridge/pen insulin now comprises over $90 \%$ of all insulin used in high-income countries, 
while in low-income countries that proportion has risen to a median of $10 \%$ (interquartile range $2-15 \%$ ) in 2009 [19].

Similarly, considerations of cost-effectiveness are also highly relevant to new treatments for people with type 2 diabetes (e.g. gliptins, glitazones, incretin mimetics). They are very costly and of unproven added value. In Kyrgyzstan, a recent RAPIA assessment found that $56 \%$ of spending on oral hypoglycaemic agents was for the purchase of repaglinide, sufficient for 3\% of the nation's type 2 diabetic patients, while only $2 \%$ was for metformin, in quantities sufficient for less than $4 \%$ of all patients [18]. None of the newer agents has been shown to influence rates of complications in type 2 diabetes, whether macrovascular or microvascular. This should be contrasted with the data on the cardiovascular benefits of metformin [32], which have contributed to this agent being widely considered as a first-line treatment in type 2 diabetes [22].

The considerations described above have prompted us to prepare the IIF Position Statement (see textbox) on treatments for people with diabetes in situations where healthcare resources are limited.

\section{Conclusions}

Financial pressures on healthcare budgets are inescapable. The setting of costs against the relative benefits of different treatments is universally considered as good practice, but the balance will clearly vary between differing socioeconomic settings. Providing the latest insulins and other diabetes treatments in resource-poor settings will divert scarce resources from more effective use in diabetes care. There are already several models of more appropriate, economical and effective deployment of resources for diabetes care in these settings. In South Africa [33] and Ghana [34] such restructuring of hospital services and educational initiatives have reduced in-patient mortality owing to hyperglycaemic emergencies. Nurse-led programmes using simple protocols and empowerment-based patient education have improved glycaemic control in people with type 2 diabetes in remote rural areas of Africa [35]. Twinning programmes between the USA and Eritrea [36], and the UK and Mozambique [15], have improved glycaemic control and/or insulin supply. In Tanzania, a

\section{Position statement on the provision and choice of diabetes treatments in resource-limited settings}

- The IIF promotes the universal access for persons with type 1 diabetes to life-saving and life-preserving insulin. The IIF also supports the availability of insulin to those type 2 diabetic persons who need insulin for optimal diabetes control and life quality.

- Insulin is an expensive drug for countries with limited healthcare resources and finances. In these countries, insulin provision may require up to $10 \%$ of the total national healthcare budget.

- Considerable cost savings may be possible by using animal (pork or beef) or biosynthetic human insulins, rather than analogue insulins. The benefits of analogue insulins are small (particularly in the absence of glucose self-monitoring) but their costs are very high. Insulin injection pens are also expensive compared with syringes and vials. Efforts should also be made to ensure that insulin is used only when necessary in type 2 diabetes.

- A wide variety of new treatments have recently become available for type 2 diabetes, e.g. glitazones, gliptins and incretin mimetics. Though useful in some patients, all of these drugs are extremely expensive and for none is there yet evidence of long-term outcome benefit. The IIF agrees that metformin and sulfonylureas should be the mainstay of drug treatment in type 2 diabetes-as recommended by the UK's Health Technology Assessment Panel and its National Institute of Health and Clinical Excellence (NICE) [22].

- Provision of diabetes education, glucose self-monitoring and expert healthcare providers are all highly important parts of the package of care for those with diabetes. More economical provision of insulin and drugs may release financing for at least some of these vital facilities.

- Diabetes drug costs can be further reduced by tendering for generic preparations from sources conforming to Good Manufacturing Practice. The introduction of a Prequalification Scheme [31], as exists to ensure quality for anti-retroviral and anti-tuberculous drugs and asthma treatments [39], would facilitate savings for health systems in many countries and should be widely encouraged. 
National Diabetes Programme has helped deliver standardised care throughout the country [37]. Even simple strategies such as moving insulin-treated patients from once daily to twice daily insulin (with no extra supportive services) has resulted in significant $\mathrm{HbA}_{1 \mathrm{c}}$ improvements in Ethiopia [38].

These and other initiatives, if widely adopted, could set the stage for greatly improved general care and control of diabetic patients in Africa and other resource-poor areas of the world. We believe that the rational use of insulin and other diabetes treatments would both aid overall insulin supply and allow better allocation of limited resources to improving systems of care for people with diabetes.

Acknowledgements The authors gratefully acknowledge the support from P. Stephens from IMS in generously providing some data for this article as well as support from the Trustees of the IFF: J. Bowis MEP, M. de Courten, A. Motala, K. Ramaiya, S. Tesfaye and N. Unwin.

Duality of interest G. Gill has received educational grants from NovoNordisk and Lilly, and research funds from Sanofi-Aventis. The IIF has previously received support for conducting RAPIAs from the World Diabetes Foundation and the International Diabetes Federation.

\section{References}

1. Chale SS, Swai AB, Mujinja PG, McLarty DG (1992) Must diabetes be a fatal disease in Africa? Study of costs of treatment. BMJ 304:1215-1218

2. Savage A (1994) The insulin dilemma: a survey of insulin treatment in the tropics. Int Diabetes Dig 5:19-20

3. Alberti K (1994) Insulin dependent diabetes mellitus: a lethal disease in the developing world. BMJ 309:754-755

4. Gill GV, Mbanya JC, Ramaiya KL, Tesfaye S (2009) A subSaharan African perspective of diabetes. Diabetologia 52:8 16

5. McLarty D, Swai ABM, Alberti KGMM (1994) Insulin availability in Africa: an insoluble problem? Int Diabetes Dig 5:15-17

6. Raab R, Fezeu L, Mbanya JC (2004) Cost and availability of insulin and other diabetes supplies: IDF survey 2002-2003. Diabetes Voice 49:24-27

7. Yudkin JS (2000) Insulin for the world's poorest countries. Lancet 355:919-921

8. Beran D, Yudkin J, de Courten M (2005) Access to care for patients with insulin-requiring diabetes in developing countries: case studies of Mozambique and Zambia. Diabetes Care 28:21362140

9. Beran D, Yudkin JS (2006) Diabetes care in sub-Saharan Africa. Lancet 368:1689-1695

10. Muyer MT, Buntinx F, Mapatano MA, De Clerck M, Truyers C, Muls E (2010) Mortality of young patients with diabetes in Kinshasa, DR Congo. Diabet Med 27:405-411

11. World Health Organization (2007) 15th Model list of essential medicines. WHO, Geneva

12. Deeb L, Tan MH, Alberti KGMM (1994) Insulin availability among International Diabetes Federation member associations. Diabetes Care 17:220-223
13. Raab R (2002) The activities of insulin for life, Australia. Diabetes Voice 47:21-23

14. Beran D, Yudkin J, de Courten M (2006) Assessing health systems for insulin-requiring diabetes in sub-Saharan Africa: developing a 'Rapid Assessment Protocol for Insulin Access'. BMC Health Serv Res 6:17

15. Beran D, Silva Matos C, Yudkin JS (2010) The Diabetes UK Mozambique Twinning Programme. Results of improvements in diabetes care in Mozambique: a reassessment 6 years later using the Rapid Assessment Protocol for Insulin Access. Diabet Med $27: 855-861$

16. International Insulin Foundation (2004) Report of the International Insulin Foundation on the Rapid Assessment Protocol for Insulin Access in Mozambique. IIF, London

17. Yudkin JS, Holt RI, Silva-Matos C, Beran D (2009) Twinning for better diabetes care: a model for improving healthcare for noncommunicable diseases in resource-poor countries. Postgrad Med J 85:1-2

18. Abdraimova A, Beran D (2009) Report on the Rapid Assessment Protocol for Insulin Access in Kyrgyzstan. IIF, London

19. IMS Health (2010) IMS Health MIDAS, London

20. Ogle G, Beran D, Raab R, Deeb L (2006) Global access and availability of insulin. Diabetes Voice 51:22-25

21. Health Action International (2010) Life-saving insulin largely unaffordable - a one day snapshot of the price of insulin across 60 countries. Available from www.haiweb.org/medicineprices/ 07072010/Global_briefing_note_FINAL.pdf, accessed 17 August 2010

22. National Collaborating Centre for Chronic Conditions (2008) Type 2 diabetes. National clinical guidance for management in promary and secondary care (update). Available from www.nice. org.uk/nicemedia/pdf/CG66FullGuideline0509.pdf, accessed 17 August 2010

23. Holleman F, Gale E (2007) Nice insulins, pity about the evidence. Diabetologia 50:1783-1790

24. Distiller LA, Joffe BI (2006) From the coalface: does glargine insulin improve hypoglycaemic episodes, glycaemic control or affect body mass in type 1 diabetic subjects who are attending a 'routine' diabetes clinic? Diabetologia 49:27932794

25. Woodward A, Weston P, Casson IF, Gill GV (2009) Nocturnal hypoglycaemia in type 1 diabetes - frequency and predictive factors. Q J Med 102:603-607

26. Cumming J, Mays N, Daube J (2010) How New Zealand has contained expenditure on drugs. BMJ 340:c2441

27. International Diabetes Federation (2008) German analogue insulin issue. Available from http://www.idf.org/German_analogue_insulin issue, accessed 15 June 2010

28. New Zealand Pharmaceutical Industry Taskforce (2007) Submission on the Towards a New Zealand Medicines Strategy Consultation Document. Available from www.rmianz.co.nz/downloads/ NZPITsub.pdf, accessed 15 June 2010

29. Volman B (2008) Direct costs and availability of diabetes medicines in low-income and middle-income countries. WHO and Health Action International, Geneva and Amsterdam

30. Currie CJ, Peters JR, Evans M (2010) Dispensing patterns and financial costs of glucose-lowering therapies in the UK from 2000 to 2008. Diabet Med 27:744-752

31. World Health Organization (2010) Prequalification programme. Available from http://apps.who.int/prequal/default.htm, accessed 4 May 2010

32. UK Prospective Diabetes Study (UKPDS) Group (1998) Effect of intensive blood-glucose control with metformin on complications in overweight patients with type 2 diabetes (UKPDS 
34). UK Prospective Diabetes Study (UKPDS) Group. Lancet 352:854-865

33. Huddle KR, Gill GV (1989) Reducing acute hyperglycaemic mortality in African diabetic patients. Diabet Med 6:64-66

34. Acheampong JW, Boateng KA, Eghan BA, Story P, Parry EHO, Tomlinson S (2000) The impact of diabetes nurses in Komfo Anokye Teaching Hospital, Ghana. Diabetes Int 10:81-93

35. Gill GV, Price C, Shandu D, Dedicoat M, Wilkinson D (2008) An effective system of nurse-led diabetes care in rural Africa. Diabet Med $25: 606-611$
36. Windus DW, Ladenson JH, Merrins CK et al (2007) Impact of a multidisciplinary intervention for diabetes in Eritrea. Clin Chem 53:1954-1959

37. Ramaiya K (2005) Tanzania and diabetes - a model for developing countries? BMJ 330:679

38. Gill GV, Gebrekidan A, English PJ, Tesfaye S (2009) Improving glycaemic control in African diabetic patients on insulin: a resource-free approach. Trop Doct 39:3-5

39. Billo N (2006) Asthma drug facility: from concept to reality. Int J Tuberc Lung Dis 10:709 